\title{
Tobacco Smoke Plays an Important Role in Initiation and Development of Lung Cancer by Promoting the Characteristics of Cancer Stem Cells
}

This article was published in the following Dove Press journal: Cancer Management and Research

\author{
Ling Lu ${ }^{\mathrm{l}, *}$ \\ Qiaoyan Liang ${ }^{2, *}$ \\ Shiyue Shen ${ }^{3}$ \\ Lei Feng ${ }^{3}$ \\ Longtao Jin' \\ Zhao feng Liang (iD ${ }^{3}$ \\ 'Women and Children Health Hospital of \\ Zhenjiang, Zhenjiang, Jiangsu 2I200I, \\ People's Republic of China; 'People's \\ Liberation Army Navy No. 97I Hospital, \\ Qingdao, Shandong 266000, People's \\ Republic of China; ${ }^{3}$ Jiangsu Key \\ Laboratory of Medical Science and \\ Laboratory Medicine, School of Medicine, \\ Jiangsu University, Zhenjiang, Jiangsu \\ 212013, People's Republic of China
}

*These authors contributed equally to this work
Correspondence: Longtao Jin Women and Children Health Hospital of Zhenjiang, 20 Zhengdong Road,

Zhenjiang, Jiangsu 21200I, People's

Republic of China

Email 13862451566@163.com

Zhao feng Liang Jiangsu Key Laboratory of Medical Science and Laboratory Medicine, School of Medicine, Jiangsu University, 30I Xuefu

Road, Zhenjiang, Jiangsu 212013, People's Republic of China

Email liangzhaofeng@ujs.edu.cn

\begin{abstract}
Lung cancer is one of the most common causes of cancer-related deaths worldwide. Tobacco smoke is the single greatest risk factor of lung cancer. Although enormous progress in understanding the molecular mechanisms by which tobacco smoke leading to lung cancer has been made, the molecular pathogenesis remains largely unclear. Cancer stem cells have been implicated in cancer initiation, development, and drug resistance. In this review, we reviewed the relationship between tobacco smoke and lung cancer, the key role of cancer stem cells in lung cancer and other tumors. More importantly, we elucidate the mechanism of tobacco smoke promoting lung cancer from the perspective of the characteristics of cancer stem cells induced by tobacco smoke.
\end{abstract}

Keywords: lung cancer, tobacco smoke, cancer stem cells, mechanism

\section{Introduction}

Lung cancer is one of the most common causes of cancer-related deaths worldwide. ${ }^{1}$ About 1.8 million people are diagnosed with lung cancer and about 1.6 million people die as a result of lung cancer annually. ${ }^{2}$ Tobacco smoke is the single greatest risk factor of lung cancer., ${ }^{3,4}$ Studies have shown that $75-90 \%$ of lung cancers are related to the use of tobacco smoke. ${ }^{4}$ Although enormous progress in understanding the molecular mechanisms by which tobacco smoke leading to lung cancer has been made, the molecular pathogenesis remains largely unclear. In this review, we attempt to elucidate the mechanism of tobacco smoke promoting lung cancer from the perspective of the characteristics of cancer stem cells promoted by tobacco smoke.

\section{Tobacco Smoke is Highly Correlated with the Initiation and Development of Lung Cancer}

Tobacco smoke is a complex chemical mixture, containing several thousand compounds, including at least 60 known carcinogens. ${ }^{5,6}$ Studies found that tobacco smoke was related to the incidence of lung cancer, and then the relationship between tobacco smoke and lung cancer has been widely studied since $1950 \mathrm{~s}$. ${ }^{5}$ It is reported that there are an estimated 1.1 billion smokers globally, 1.8 million deaths from lung cancer each year, and about $80-90 \%$ of those deaths are attributable to tobacco smoke exposure. ${ }^{6,7}$ More and more evidences show that tobacco smoke promote the occurrence and development of lung cancer. ${ }^{6-10}$ It is reported 
that smokers have up to a 30-fold higher risk of developing lung cancer than non-smokers. ${ }^{6,7}$ Tobacco smoke is a tumor initiator and a tumor promoter. ${ }^{9}$ Conversion of tobacco smoke compounds to reactive forms results in gene mutation, DNA damage, abnormal DNA methylation, formation of DNA adducts, pulmonary inflammation, oxidative stress, abnormal proliferation, abnormal differentiation, Epithelial-Mesenchymal Transition (EMT) and so on. ${ }^{7-9,11}$

Enormous progress in understanding the molecular mechanisms by which tobacco smoke leading to lung cancer has been made; however, the molecular pathogenesis remains largely unclear. More and more studies have found that cancer stem cells (CSCs) play key roles in cancer initiation, development and relapse. ${ }^{12}$ Herein, this article focuses on tobacco smoke affecting CSCs, thereby promoting the occurrence and development of lung cancer.

\section{Cancer Stem Cells Play a Key Role in Tumorigenesis and Development}

It is believed that CSCs are responsible for cancer initiation and local or metastatic recurrence. It may be because the inherent self-renewal and tumorigenic properties of these cells provide them with unique capabilities to resist various forms of anti-cancer treatment, disseminate to and colonize distant tissues. ${ }^{12}$ CSCs are resistant to conventional chemotherapy and radiation therapy, and can remain dormant for many years before cancer initiation, metastases and relapses. ${ }^{13}$ Some previous studies showed that the critical role of CSCs in initiation and development of lung cancer, liver cancer, breast cancer, head and neck squamous cell carcinomas, pancreatic cancer and glioblastoma. $^{14-19}$

Lung CSCs are considered as the cancer initiating and maintaining cells of lung cancer. ${ }^{15}$ Lin et al reported that lung CSCs are responsible for lung cancer initiation and local or metastatic recurrence, the mitochondrial deoxyguanosine kinase is required for CSCs stemness in lung adenocarcinoma. ${ }^{13}$ Wang et al investigated that arsenic and $\mathrm{BaP}$ co-exposure produce CSC-like cells and promote the occurrence of lung cancer. ${ }^{20}$ Hsu et al reported that lung CSCs confer tumorigenesis and resistance to standard treatment in lung cancer, targeting the upregulated glycolysis and its associated downstream pathways may be the novel strategies for eliminating CSCs and the better prognosis of cancer. $^{21}$ These studies have shown that lung
CSCs play a very important role in the occurrence, development and treatment of lung cancer.

\section{Tobacco Smoke Enhance the Stemness of Cancer Stem Cells}

Toxicants, such as tobacco smoke, have various enhancement effects on cancer stem cells. Acquisition of CSCs properties induced by tobacco smoke is an early event in the initiation of carcinogenesis. Xie et al reported that long-term tobacco smoke exposure increased the stemness of hepatic CSCs, induced EMT process and IL-33 expression via IL-33/p38. ${ }^{22}$ Liang et al found that long time tobacco smoke exposure induced urocystic EMT and acquisition of CSCs properties by activating the Wnt pathway. ${ }^{23}$ Tobacco smoke is a major risk factor for pancreatic cancer and exposure to tobacco smoke activates stem cell features of pancreatic cancer cells via PAF $1 .^{24}$ Qian et al found that tobacco smoke effectively promoted renal CSCs stemness via Sonic Hedgehog pathway by enhancing self-renewal ability, increasing the expression of CSCs markers and elevating $\mathrm{CD}_{133^{+}}$cell population. ${ }^{25}$ It is reported that nicotine is effective in the occurrence, the development and metastasis of breast cancer by inducing enrichment of side population cells with CSCs-like properties. $^{26,27}$ Exposure to chewing tobacco smoke increased cell proliferation, invasive ability and anchorage independent growth. Datta et al proposed that chronic exposure of esophageal epithelial cells to tobacco smoke leads to CSCs-like phenotype. ${ }^{28}$ In summary, tobacco smoke enhanced the self-renewal ability, stemness, and induce the generation of CSCs-like cells, which promoted the occurrence and development of liver cancer, kidney cancer, breast cancer, etc.

More importantly, it is believed that tobacco smoke is effective in the occurrence, the development and metastasis of lung cancer by enhancing the stemness of lung CSCs (Figure 1). Schaal et al demonstrated that nicotine can induce the expression of Sox 2 and Oct4, which are indispensable for self-renewal and maintenance of CSCs properties in non-small cell lung adenocarcinoma cells. ${ }^{29} \mathrm{Xie}$ et al suggested that long-term tobacco smoke exposure enhancing self-renewal ability, increasing the expression of CSCs markers and tumorigenic ability of human bronchial epithelial cells, the IL- $6 / \Delta \mathrm{Np} 63 \alpha /$ Notch axis and $\mathrm{Wnt} / \beta$-catenin pathway play an important role in this process. $^{30,31}$ Mouse models with initial and advanced forms of lung cancer were developed using tobacco 


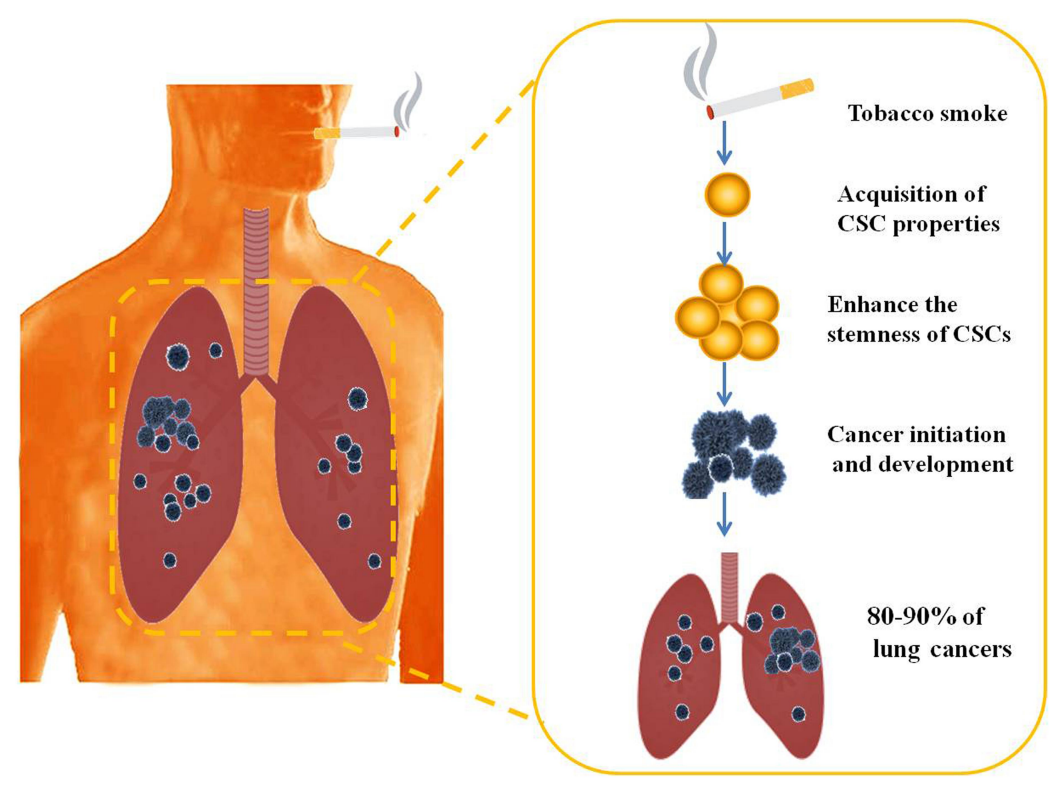

Figure I Tobacco smoke is effective in the occurrence and development of lung cancer by enhancing the stemness of lung CSCs.

smoke carcinogen, the number of OCT4-positive cells increases as lung cancer progresses and play important role in lung cancer progresses. ${ }^{32}$ Experiments by Liu et al have shown that long-term tobacco smoke exposure promote formation of CSCs, EMT process and malignant transformation. ${ }^{33}$ Wang et al demonstrated that epigenetic silencing of miR-218 is involved in the acquisition of CSC-like properties and malignant transformation of human bronchial epithelial cells induced by long-term tobacco smoke exposure. ${ }^{34}$ Tobacco smoke could induce the generation of lung CSCs and enhance the self-renewal ability and stemness of lung CSCs, which plays a key role in the occurrence and development of lung cancer. These findings may have important significance in the prevention, diagnosis and treatment of lung cancer.

\section{Summary and the Future Challenges}

Based on the above research, we found that tobacco smoke promotes the acquisition of CSCs characteristics and enhanced CSCs properties, then play an important role in the process of lung cancer initiation, development, metastasis, etc. However, there are still many unknown need to study and explore, and many improvement and challenges in the future research.

Excellent and highly recognized cell and animal models need development and establishment. Based on these excellent models, we can use new techniques to study the exact role and molecular mechanism of tobacco smoke- induced CSCs in the initiation, development of lung cancer. Then, these data will provide theoretical basis for targeted therapy and precise treatment.

\section{Funding}

This work was supported by guiding project of social development in Zhenjiang (No.FZ2019038), the Foundation for Excellent Young Teachers of Jiangsu University, Research and Practice innovation program for graduate students of Jiangsu Province (No.KYCX20_3089) and the Student Research Project of Jiangsu University, Student Scientific Research Project of Jiangsu University (No.18A451, 18A474).

\section{Disclosure}

Ling Lu and Qiaoyan Liang shared equal contribution and are co-first authors for this study. All authors of this study declare no conflicts of interest for this work.

\section{References}

1. Pankova D, Jiang Y, Chatzifrangkeskou M, et al. RASSF1A controls tissue stiffness and cancer stem-like cells in lung adenocarcinoma. EMBO J. 2019;38(13):e100532. doi:10.15252/embj.2018100532

2. Hirsch FR, Scagliotti GV, Mulshine JL, et al. Lung cancer: current therapies and new targeted treatments. Lancet. 2017;389 (10066):299-311. doi:10.1016/S0140-6736(16)30958-8

3. Sheng L, Tu JW, Tian JH, Chen HJ, Pan CL, Zhou RZ. A meta-analysis of the relationship between environmental tobacco smoke and lung cancer risk of nonsmoker in China. Medicine. 2018;97(28):e11389. doi:10.1097/MD.0000000000011389

4. de Groot PM, Wu CC, Carter BW, Munden RF. The epidemiology of lung cancer. Transl Lung Cancer Res. 2018;7(3):220-233. doi:10.21037/tlcr.2018.05.06 
5. Lu L, Xu H, Yang P, et al. Involvement of HIF-1 $\alpha$-regulated miR-21, acting via the Akt/NF- $\mathrm{kB}$ pathway, in malignant transformation of HBE cells induced by cigarette smoke extract. Toxicol Lett. 2018;289:14-21. doi:10.1016/j.toxlet.2018.02.027

6. Yoshida K, Gowers KHC, Lee-Six H, et al. Tobacco smoking and somatic mutations in human bronchial epithelium. Nature. 2020;578 (7794):266-272. doi:10.1038/s41586-020-1961-1

7. Pfeifer GP. Smoke signals in the DNA of normal lung cells. Nature. 2020;578(7794):224-226. doi:10.1038/d41586-020-00165-7

8. Vaz M, Hwang SY, Kagiampakis I, et al. Chronic cigarette smoke-induced epigenomic changes precede sensitization of bronchial epithelial cells to single-step transformation by KRAS mutations. Cancer Cell. 2017;32 (3):360-376 e366. doi:10.1016/j.ccell.2017.08.006

9. Takahashi H, Ogata H, Nishigaki R, Broide DH, Karin M. Tobacco smoke promotes lung tumorigenesis by triggering IKK $\beta$ - and JNK1dependent inflammation. Cancer Cell. 2010;17(1):89-97. doi:10. 1016/j.ccr.2009.12.008

10. Ahluwalia IB, Smith T, Arrazola RA, et al. Current tobacco smoking, quit attempts, and knowledge about smoking risks among persons aged $>/=15$ years - global adult tobacco survey, 28 countries, 2008-2016. MMWR Morb Mortal Wkly Rep. 2018;67 (38):1072-1076. doi:10.15585/mmwr.mm6738a7

11. Goldkorn T, Filosto S, Chung S. Lung injury and lung cancer caused by cigarette smoke-induced oxidative stress: molecular mechanisms and therapeutic opportunities involving the ceramide-generating machinery and epidermal growth factor receptor. Antioxid Redox Signal. 2014;21(15):2149-2174. doi:10.1089/ars.2013.5469

12. Clara JA, Monge C, Yang Y, Takebe N. Targeting signalling pathways and the immune microenvironment of cancer stem cells a clinical update. Nat Rev Clin Oncol. 2020;17(4):204-232.

13. Lin S, Huang C, Sun J, et al. The mitochondrial deoxyguanosine kinase is required for cancer cell stemness in lung adenocarcinoma. $E M B O M O l$ Med. 2019;11(12):e10849. doi:10.15252/emmm.201910849

14. Huang C, Yoon C, Zhou XH, et al. ERK1/2-Nanog signaling pathway enhances CD44(+) cancer stem-like cell phenotypes and epithelial-tomesenchymal transition in head and neck squamous cell carcinomas. Cell Death Dis. 2020;11(4):266. doi:10.1038/s41419-020-2448-6

15. Liu P, Zhang R, Yu W, et al. FGF1 and IGF1-conditioned 3D culture system promoted the amplification and cancer stemness of lung cancer cells. Biomaterials. 2017;149:63-76. doi:10.1016/j. biomaterials.2017.09.030

16. Zhang C, Huang S, Zhuang H, et al. YTHDF2 promotes the liver cancer stem cell phenotype and cancer metastasis by regulating OCT4 expression via m6A RNA methylation. Oncogene. 2020;39 (23):4507-4518. doi:10.1038/s41388-020-1303-7

17. Huang P, Liao R, Chen X, et al. Nuclear translocation of PLSCR1 activates STAT1 signaling in basal-like breast cancer. Theranostics. 2020;10(10):4644-4658. doi:10.7150/thno.43150

18. Alcala S, Sancho P, Martinelli P, et al. ISG15 and ISGylation is required for pancreatic cancer stem cell mitophagy and metabolic plasticity. Nat Commun. 2020;11(1):2682. doi:10.1038/s41467-020-16395-2

19. Buccarelli M, Lulli V, Giuliani A, et al. Deregulated expression of the imprinted DLK1-DIO3 region in glioblastoma stem-like cells: tumor suppressor role of lncRNA MEG3. Neuro-Oncology. 2020. doi:10.1093/neuonc/noaa127

20. Wang Z, Yang P, Xie J, et al. Arsenic and benzo[a]pyrene co-exposure acts synergistically in inducing cancer stem cell-like property and tumorigenesis by epigenetically down-regulating SOCS3 expression. Environ Int. 2020;137:105560. doi:10.1016/j.envint.2020.105560
21. Hsu HS, Liu CC, Lin JH, et al. Involvement of collagen XVII in pluripotency gene expression and metabolic reprogramming of lung cancer stem cells. J Biomed Sci. 2020;27(1):5. doi:10.1186/s12929-019-0593-y

22. Xie C, Zhu J, Wang X, et al. Tobacco smoke induced hepatic cancer stem cell-like properties through IL-33/p38 pathway. J Exp Clin Cancer Res. 2019;38(1):39. doi:10.1186/s13046-019-1052-Z

23. Liang Z, Lu L, Mao J, Li X, Qian H, Xu W. Curcumin reversed chronic tobacco smoke exposure induced urocystic EMT and acquisition of cancer stem cells properties via Wnt $/ \beta$-catenin. Cell Death Dis. 2017;8(10):e3066. doi:10.1038/cddis.2017.452

24. Nimmakayala RK, Seshacharyulu P, Lakshmanan I, et al. Cigarette smoke induces stem cell features of pancreatic cancer cells via PAF1. Gastroenterology. 2018;155(3):892-908 e896. doi:10.1053/j. gastro.2018.05.041

25. Qian W, Kong X, Zhang T, et al. Cigarette smoke stimulates the stemness of renal cancer stem cells via Sonic Hedgehog pathway. Oncogenesis. 2018;7(3):24. doi:10.1038/s41389-018-0029-7

26. Guha P, Bandyopadhyaya G, Polumuri SK, et al. Nicotine promotes apoptosis resistance of breast cancer cells and enrichment of side population cells with cancer stem cell-like properties via a signaling cascade involving galectin-3, $\alpha 9$ nicotinic acetylcholine receptor and STAT3. Breast Cancer Res Treat. 2014;145(1):5-22. doi:10.1007/ s10549-014-2912-z

27. Turker Sener L, Guven C, Sener A, Adin Cinar S, Solakoglu S, Albeniz I. Nicotine reduces effectiveness of doxorubicin chemotherapy and promotes CD44(+)CD24(-) cancer stem cells in MCF-7 cell populations. Exp Ther Med. 2018;16(1):21-28.

28. Datta KK, Patil S, Patel K, et al. Chronic exposure to chewing tobacco induces metabolic reprogramming and cancer stem cell-like properties in esophageal epithelial cells. Cells. 2019;8(9):949. doi:10.3390/cells 8090949

29. Schaal CM, Bora-Singhal N, Kumar DM, Chellappan SP. Regulation of Sox 2 and stemness by nicotine and electronic-cigarettes in non-small cell lung cancer. Mol Cancer. 2018;17(1):149. doi:10.1186/s12943-018-0901-2

30. Xie C, Zhu J, Jiang Y, et al. Sulforaphane inhibits the acquisition of tobacco smoke-induced lung cancer stem cell-like properties via the IL-6/deltaNp63alpha/notch axis. Theranostics. 2019;9 (16):4827-4840. doi:10.7150/thno.33812

31. Wang J, Chen J, Jiang Y, et al. Wnt $/ \beta$-catenin modulates chronic tobacco smoke exposure-induced acquisition of pulmonary cancer stem cell properties and diallyl trisulfide intervention. Toxicol Lett. 2018;291:70-76. doi:10.1016/j.toxlet.2018.04.003

32. Ao X, Zhou J, Liang HL, Jiang M, Li HS. Expression profile of Oct4lung cancer-specific marker prior and subsequent to a salirasib treatment regime. Oncol Lett. 2017;14(5):5145-5148.

33. Liu Y, Luo F, Xu Y, et al. Epithelial-mesenchymal transition and cancer stem cells, mediated by a long non-coding RNA, HOTAIR, are involved in cell malignant transformation induced by cigarette smoke extract. Toxicol Appl Pharmacol. 2015;282(1):9-19. doi:10.1016/j.taap.2014.10.022

34. Wang B, Liu Y, Luo F, et al. Epigenetic silencing of microRNA-218 via EZH2-mediated $\mathrm{H} 3 \mathrm{~K} 27$ trimethylation is involved in malignant transformation of HBE cells induced by cigarette smoke extract. Arch Toxicol. 2016;90(2):449-461. doi:10.1007/s00204-014-1435-z 


\section{Publish your work in this journal}

Cancer Management and Research is an international, peer-reviewed open access journal focusing on cancer research and the optimal use of preventative and integrated treatment interventions to achieve improved outcomes, enhanced survival and quality of life for the cancer patient.

The manuscript management system is completely online and includes a very quick and fair peer-review system, which is all easy to use. Visit http://www.dovepress.com/testimonials.php to read real quotes from published authors.

Submit your manuscript here: https://www.dovepress.com/cancer-management-and-research-journal 